\title{
MicroRNA-1 inhibits the proliferation of chinese sika deer-derived cartilage cells by binding to the 3 '-untranslated region of IGF-1
}

\author{
WEI HU, XINGYU MENG, TIANCHENG LU, LEI WU, TING LI, MU LI and YUHUA TIAN \\ Department of Biochemistry and Molecular Biology, College of Life Sciences, \\ Jilin Agriculture University, Changchun, Jilin 130118, P.R. China
}

Received January 21, 2013; Accepted May 24, 2013

DOI: $10.3892 / \mathrm{mmr} .2013 .1515$

\begin{abstract}
Insulin-like growth factor-1 (IGF-1) is critical in the proliferation and regeneration of Chinese sika deer antler cells. The regulation of IGF-1 is complex and remains unclear. In the present study, miRNA GeneChip ${ }^{\circledR}$ and TargetScan Human software were used to identify microRNA-1 (miR-1), which binds to the 3'-untranslated region (3'UTR) of IGF-1. An miR-1 mimic was transfected into antler cartilage cells in order to induce the overexpression of miR-1. The expression levels of miR-1 in antler cartilage cells were determined by quantitative polymerase chain reaction (qPCR). A high-throughput luciferase reporter screen was used to demonstrate the potential regulation of IGF-1 by miR-1. miR-1 suppressed the luciferase activity of the pmiR-IGF-1 by 50\% compared with the negative control (NC). An MTT assay and cell cycle analyses confirmed that the overexpression of miR-1 significantly inhibited the proliferation of cartilage cells $(\mathrm{P}<0.05)$. Furthermore, western blot analysis revealed that overexpressed miR-1 downregulated the protein levels of IGF-1. The 3'UTR of IGF-1 was found to have an miR-1 combining site, which allowed the inhibition of IGF-1 protein expression, as demonstrated by a luciferase reporter assay, and miR-1 was shown to be an important and effective means of regulating IGF-1. In conclusion, miR-1 downregulated the protein expression of IGF-1 by directly targeting the 3'UTR of IGF-1. miR-1 may be crucial for inhibiting the proliferation of deer antler cartilage cells. Our findings provided the evidence for the first time that miR-1 directly regulates the expression of IGF-1 by directly targeting its 3'UTR.
\end{abstract}

Correspondence to: Dr Wei Hu, Department of Biochemistry and Molecular Biology, College of Life Sciences, Jilin Agriculture University, 2888 Xincheng Street, Changchun, Jilin 130118, P.R. China

E-mail: huwei9002@126.com

Key words: sika deer, regeneration, microRNA, insulin-like growth factor-1, cartilage cells, proliferation

\section{Introduction}

Several studies have proposed that the regeneration and growth of deer antlers may be used as a model for biomedical research $(1,2)$. Deer antlers are bony organs, which are cast and regenerated each year. The antler growth center is located in its tip and the antler consists of three layers. These layers are distoproximally the reserve mesenchyme, precartilage and cartilage. Antler growth is achieved through the activity of cells residing in the proliferation zone, particularly in the reserve cartilage layer. Insulin-like growth factor-1 (IGF-1) is a multifunction cell proliferation regulation factor, named due to its structural similarity to insulin (3). Numerous studies have observed that the biological activation of IGF-1 is varied, playing an active role in cell growth, embryonic differentiation, metabolism and the adjustment of the nervous and endocrine systems (4-6). IGF-1 has a positive effect on controlling cell metabolism, regulating cell division and differentiation, inhibiting cell apoptosis and various cell function adjustments (7).

The proliferation of deer antler cells may be regulated by IGF-1, which has a major effect on promoting the formation of cartilage (8). As IGF-1 has a growth-promoting role in cartilage formation and the majority of the antler growth center is composed of cartilage, Suttie et al (8) suggested that IGF-I may be the antler growth stimulus (AGS). IGF-1 receptors are located in growing antler tissues (9). Results of a study by Sadighi et al (10) support the theory that IGF-1 may be the AGS. IGF-1 was found to increase the proliferation of mesenchymal and cartilaginous cells derived from the antler proliferation zone in a dose-dependent manner. The development of deer antlers and the concentration of IGF-1 have a dependent relationship, such that regions containing high levels of IGF-1 grow rapidly (11). The content of IGF-1 gradually decreases from the top to the bottom of the antler; the cartilage layer has the highest level of IGF-1 and the mesenchyme layer has the lowest level of IGF-1. This is consistent with the theory that cartilage is the fastest growing area of the deer antler (12). However, the underlying mechanisms regulating the rapid proliferation of velvet antler remain unclear.

MicroRNAs (miRNAs) are a class of endogenous non-coding RNAs formed of 18-24 nucleotides, whose function is to downregulate or inhibit the translation of mRNA and to control gene expression through binding to the 3'-untranslated region (3'UTR) of mRNA (13). Since miRNA 
was first located in Caenorhabditis elegans in 1993, it has been studied in various biological processes (14). With the development of research, a large number of miRNAs have been identified and are known to widely exist in numerous plants and animals (15). According to the MiRBase database, $>9,000$ miRNAs have been found in eukaryotes, including 1,000 miRNAs in humans (16). Furthermore, information in the MiRBase database (www.MiRBase.org) suggests that a single miRNA may regulate various target genes, different miRNAs are capable of regulating the same target genes and $\sim 30 \%$ of protein-coding genes may be regulated by miRNAs (17). Since the diversity and universality of miRNAs have been revealed, certain scholars have hypothesized that miRNA molecules may be the core component of the gene regulation network (18). Numerous studies investigating miRNA have revealed a deeper gene expression regulation system in organisms, and highlighted the important role of RNA in network control systems (19). However, whether miRNA is deregulated during the proliferation of deer antler cells and its role in progression remains unclear.

In the present study, we investigated whether IGF-1 is regulated by miRNAs. We used miRNA GeneChip ${ }^{\circledR}$ (Affymetrix, Santa Clara, CA, USA) and an miRNA target-prediction tool to identify miRNAs that may target IGF-1. MicroRNA-1 (miR-1) was identified as participating in the growth of antler tissues and the restoration of miR-1 expression in deer antler cell lines reduced cell proliferation. Furthermore, we found that the effects of miR-1 were achieved by binding directly to the 3'UTR of IGF-1, thus inhibiting the expression of IGF-1. Our results suggest that miR-1 is crucial in the proliferation of deer antler cells and has potential applications with regard to antler regeneration and growth.

\section{Materials and methods}

Cell culture. Top tissue samples from deer antlers were obtained from a three-year-old male Cervus nippon (Changchun, China). Mesenchyme and cartilage tissues were isolated under a dissecting microscope. The tissues were blended and digested by collagenase I and hyaluronic acid (Sigma-Aldrich, St. Louis, MO, USA) for $1.5 \mathrm{~h}$ at $37^{\circ} \mathrm{C}$, respectively. Subsequently, the tissues were digested further by adding collagenase-II (Sigma-Aldrich) for $3 \mathrm{~h}$ at $37^{\circ} \mathrm{C}$. Cartilage cells were cultured in DMEM (HyClone, Logan, UT, USA) with $20 \%$ fetal bovine serum (FBS), $200 \mathrm{U} / \mathrm{ml}$ penicillin and $100 \mathrm{U} / \mathrm{ml}$ streptomycin (Tianjin Hao Yang Biological Technology Co., Ltd. Tianjin, China) at $37^{\circ} \mathrm{C}$ with $5 \% \mathrm{CO}_{2}$. The study was approved by the ethics committee of the Institute of Life Sciences, Jilin Agriculture University, Changchun, China.

miRNA gene chip preparation. After culturing mesenchyme cells and cartilage cells for $72 \mathrm{~h}$, they were centrifuged and washed with PBS, respectively. miRNA gene chips were synthesized by RiboBio Co., Ltd. (Guangzhou, China). Data were analyzed using miRNA QC Tool 1 software (Beijing Boao Biological Co., Ltd., Beijing, China) in order to detect differentially expressed miRNAs in the top tissue of the deer antler.

Transfection. Cartilage cells were seeded into each well of 96-well or 6-well plates, respectively. Cells were incubated overnight and subsequently transfected with negative scramble control (NC) RNA or an miR-1 mimic using X-tremeGENE HP DNA transfection reagent (Roche, Mannheim, Germany) at a final concentration of $50 \mathrm{nM}$.

Quantitative polymerase chain reaction ( $q P C R)$. Following transfection with miR-1 for 24 and $48 \mathrm{~h}$, total RNA, including miRNA, was isolated as described previously (12) and subsequently reverse transcribed to cDNA with a stem-loop RT primer for miR-1 and Oligo $\mathrm{d}(\mathrm{T})_{18}$ for the $\beta$-actin gene. The stem-loop RT primer for miR-1 analysis was 5'-ATGACTGGCCTCCTCAGATCAGTTTGGCCACTG ACTGATCTGAAGGCCAGTCATATACATACTTCTTTA CATTCCA-3' and the qPCR primers were 5'-GCATCTCCA GCCTCCTCAGAT-3' and 5'-GCGCTGGAATGTAA AGAAGTATGTAT-3'. The PCR primers for the $\beta$-actin internal control were 5'-TGACCCTTAAGTACC CCATCGA-3' and 5'-TTGTAGAAGGTGTGGTGCCAGAT-3'. Relative expression levels were calculated using the $2^{-\Delta \Delta \mathrm{Ct}}$ method.

Cell proliferation assays. Deer antler cartilage cells, seeded into each well of 96-well plates with complete medium, were transfected with an miR-1 mimic (50 $\mathrm{nM})$ using the $\mathrm{X}$-tremeGENE HP DNA transfection reagent. The control group did not require transfection. Following culture for 24 and $48 \mathrm{~h}$, cell growth was measured using the MTT method. Spent cell culture medium was replaced with $0.1 \mathrm{ml}$ of fresh medium containing $0.5 \mathrm{mg} / \mathrm{ml}$ MTT. Cells were incubated at $37^{\circ} \mathrm{C}$ for $4 \mathrm{~h}$ and the blue-violet crystal precipitate was resolved with $0.1 \mathrm{ml}$ DMSO (Sigma-Aldrich). Absorbance was measured at $570 \mathrm{~nm}$.

Detection of cell cycle. At 24 and 48 h post-transfection, the cartilage cells were harvested and resuspended in PBS, fixed with $75 \%$ ice-cold ethanol at $4{ }^{\circ} \mathrm{C}$ overnight and stained using a $10 \%$ RNase A and $4 \%$ propidium iodide (PI) kit (Beijing Dingguo Biotechnology Co., Ltd., Beijing, China) for $1 \mathrm{~h}$ at $37^{\circ} \mathrm{C}$. Stained cells were detected using flow cytometry (FCM) and the data were analyzed using Cell-Quest software (Becton Dickinson, San Jose, CA, USA).

IGF-1 3'UTR luciferase reporter assay. The acquired 3'UTR sequence of IGF-1 was cloned into the pmiR-RB-Report ${ }^{\mathrm{TM}}$ vector, downstream of the cytomegalovirus promoter-driven firefly luciferase cassette, in order to construct luciferase reporters with the 3'UTR of IGF-1. Cells were transiently transfected with $50 \mathrm{ng}$ of luciferase reporter plasmid and $5 \mathrm{pmol}$ of miR-1 mimic using X-tremeGENE HP DNA transfection reagent. Following culture for 24 and $48 \mathrm{~h}$, the cell proteins were extracted. Luciferase activity was measured using the Dual-Luciferase Reporter Assay system (Promega, Madison, WI, USA), according to the manufacturer's instructions. Data were normalized by Photinus luciferase activity.

Western blot analysis. Total protein was extracted from cells using RIPA lysis buffer with $1 \mathrm{mM}$ phenylmethyl sulfonylfluoride at $0^{\circ} \mathrm{C}$. Following mixing with a loading buffer, the protein was separated by $12 \%$ SDS-PAGE and transferred to polyvinylidene fluoride membranes. Non-specific binding was blocked with $5 \%$ dried skim milk in a Tris-buffered saline 
and Tween-20 (TBST) mixture for 2 h. Subsequently, the membranes were incubated with primary antibodies against IGF-1 at a 1:500 dilution and GAPDH at a 1:1,000 dilution at $4^{\circ} \mathrm{C}$ overnight. Primary antibody was rabbit polyclonal anti-IGF1R antibody or rabbit polyclonal anti-GAPDH antibody. Following washing with TBST three times, the membranes were incubated with the secondary antibody goat anti-rabbit IgG-HRP conjugated at a 1:2,000 dilution at $25^{\circ} \mathrm{C}$ for $2 \mathrm{~h}$. Following washing with TBST a further three times, immunoreactive bands were visualized using enhanced chemiluminescence (ECL) reagents (Beijing Kang for Century Biotechnology Co. Ltd., Beijing, China).

Statistical analysis. The data are presented as the mean \pm SD. SPSS Statistics 12.0 software was used for all statistical analyses. $\mathrm{P}<0.05$ was considered to indicate a statistically significant difference.

\section{Results}

miRNA screening targeting the IGF-1 3'UTR. According to the analysis of $>5,000$ miRNAs by the Affymetrix miRNA GeneChip technology, we identified 210 miRNAs that exhibited a marked differential expression in the mesenchyme and cartilage cells. Compared with the mesenchyme cells, 126 miRNAs were upregulated in the cartilage cells and 84 were downregulated. Furthermore, miRNA binding site analysis of the 3'UTR of IGF-1 by TargetScan Human 5.1 software (http://www.targetscan.org) revealed that 25 miRNAs may target the 3'UTR of IGF-1 and inhibit its expression. In order to further investigate the mechanisms responsible for the promotion of antler regeneration by miR-1, we sought to identify the molecular targets of miR-1, since miRNAs function through the post-transcriptional inhibition of their target mRNAs by binding to the 3'UTR. Among the numerous predicted target genes in TargetScan, the TargetScan prediction indicated that the deer IGF-1 3'UTR contained a conserved putative miR-1 target site. The characteristics of miR-1 conformed to the results of gene chip screening and software analysis for the deer IGF-1 (Fig. 1). miR-1 was used in subsequent experiments.

Detection of miR-1 expression levels by qPCR. Subsequently, we further determined the effects of miR-1 on cartilage cells. qPCR analysis revealed that the expression levels of miR-1 in transfected cells were significantly increased compared with the untransfected normal cells, where $\beta$-actin was used as an internal control. As shown in Fig. 2, miR-1 expression levels decreased after $48 \mathrm{~h}$, and levels in transfected cells decreased after $24 \mathrm{~h}$. This indicated that miR-1 had been successfully transfected into the cartilage cells and subsequent tests were performed.

Restoration of miR-1 inhibits cartilage cell growth. During the investigation of the molecular mechanisms for miR-1-induced IGF-1 inhibition, the expression of miR-1 was found to be inversely correlated with cellular IGF-1 levels. IGF-1 has an important role in antler cell growth. We sought to determine whether miR-1 affects the proliferation rate of cartilage cells using an MTT assay and cell cycle analysis. Cell growth curves

\section{Conserved miR-1 target site in IGF-1 3'UTR}

$\begin{array}{ll}\text { Human } & \text { 5'-CCAAUGAAAUACACAAGUAAACAUUCCAA-3'(165-193) } \\ \text { Mouse } & \text { 5'-CCAAUGAAAUAUACAAGUAAACAUUCCAA-3' } \\ \text { Rat } & \text { 5'-UCAAUGAAAUAUACAAGUAAACAUUCCAA-3' } \\ \text { Cow } & \text { 5'-UCCCCCAAUGAAAUAAGUAAACAUUCCAA-3', } \\ \text { Deer } & \text { 5'-CCCCCCAGUGAAACGAGUAAACAUUCCAA-3' } \\ \text { miR-1 } & \text { 3'-UAUGUAUGAAGAAAUGUAAGGU-5' }\end{array}$

Figure 1. miRNA screening targeting insulin-like growth factor-1 (IGF-1) in the 3'-untranslated region (3'UTR). An miRNA gene chip was used to determine the miRNA content in the cartilage and mesenchyme cells. Ratios of miRNA content between the two cell types that were more than double or less than half were considered to indicate differential expression. In Chinese sika deer, IGF-1 may be a molecular target of miR-1. This figure shows the sequence alignment of miR-1 and its conserved target site in the 3'UTR of IGF-1, which was downloaded from TargetScan. Predicted miR-1 target sequences in the 3'UTR region of IGF-1 are presented. As predicted, two sections of miR-1 bind, respectively. miRNA, microRNA; miR-1, microRNA-1.

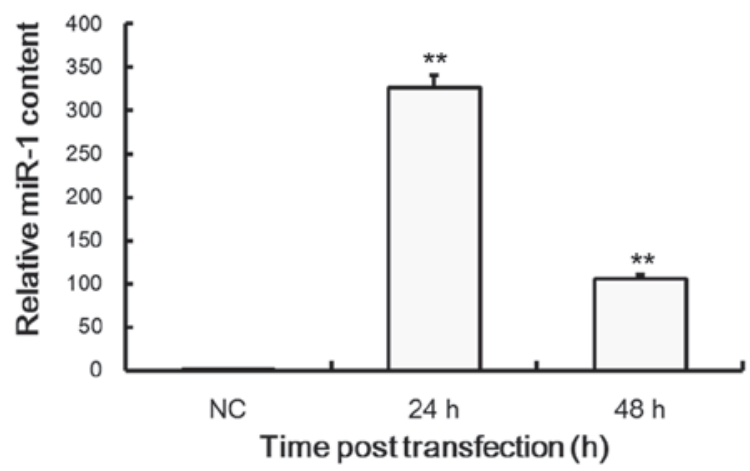

Figure 2. mir-1 content increased markedly following transfection. The relative quantitative results for the content of miR-1 in cartilage cells following transfection for 24 and $48 \mathrm{~h}$ are shown. $\beta$-actin was used as a loading control. The content of miR-1 in the cartilage cells increased markedly compared with the control cells and untransfected miR-1 mimics. Data are presented as the mean $\pm S D(n=3)$. Similar results were found in three independent experiments. ${ }^{* *} \mathrm{P}<0.01$ compared with $\mathrm{NC}$. NC, negative scramble control; miR-1, microRNA-1.

demonstrated that the overexpression of miR-1 significantly suppressed cartilage cell proliferation in a time-dependent manner when compared with the NCs, and this suppression occurred within $48 \mathrm{~h}$ in the cartilage cells $(\mathrm{P}<0.05$; Fig. 3A). To further determine the effect of miR-1 on cartilage cells, cell cycle analysis was performed. As shown in Fig. 3B and C, compared with the NCs, miR-1 induced a significant decrease in the cell cycle process in the cartilage cells. Compared with the control group, the number of cells at the $G_{1}$ and $G_{2}$ phases were markedly increased. Furthermore, the number of cells at the $\mathrm{S}$-phase decreased $(\mathrm{P}<0.05$, Fig. $3 \mathrm{~B})$. Taken together, these results demonstrated that miR-1 acted as an inhibitor and was capable of markedly reducing the proliferation of antler cells.

miR-1 regulates $I G F-1$ by binding to its 3 'UTR. miRNAs regulate the expression of target genes by binding to the 3'UTR of specific mRNAs, triggering mRNA degradation or translational repression (14). To confirm whether IGF-1 is a direct target of miR-1 in cartilage cells, a 510 bp fragment of the 3'UTR of IGF-1 containing the target sequence was cloned 

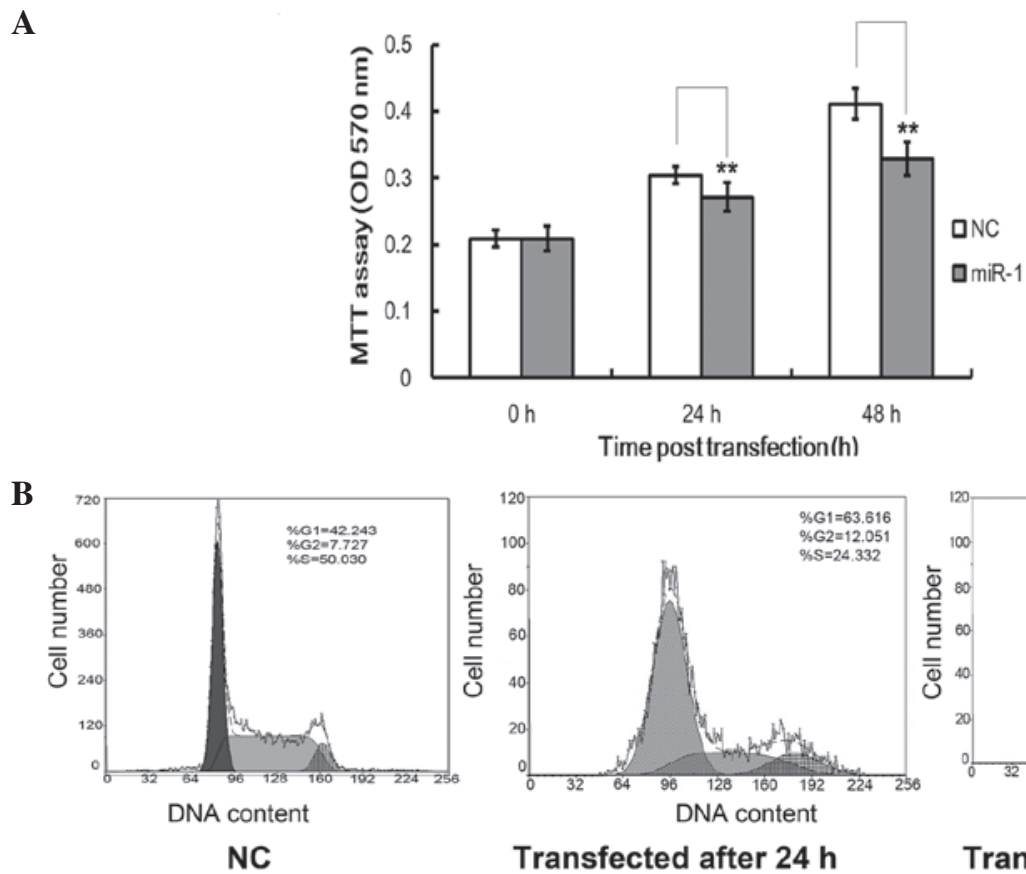

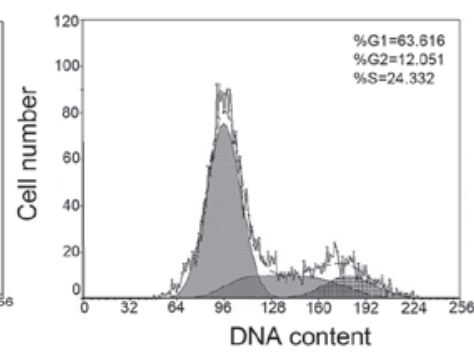

Transfected after $24 \mathrm{~h}$

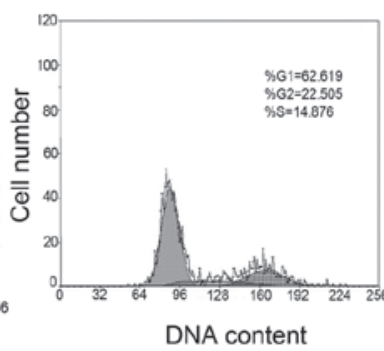

Transfected after $48 \mathrm{~h}$

Figure 3. miR-1 inhibits cartilage cell growth. (A) Cartilage cells were transfected with the negative scramble control (NC) or microRNA-1 (miR-1) mimic as indicated. The rate of cell inhibition was measured at the indicated times post-transfection using an MTT assay. Compared with the NCs, cell inhibition decreased after 24 and $48 \mathrm{~h}$, respectively. Data are presented as the mean $\pm \mathrm{SD}(\mathrm{n}=3)$. Similar results were found in three independent experiments. ${ }^{* *} \mathrm{P}<0.01$, compared with NC. (B) After $48 \mathrm{~h}$, the cultured cell medium was replaced with fresh serum-free medium. Cell cycles were detected at the indicated times post-serum deprivation. Cell cycle analysis revealed that miR-1 prevented cartilage cells from entering the S-phase and the cell percentage of $\mathrm{G}_{1} / \mathrm{G}_{2}$ was higher than that of the NCs.

A

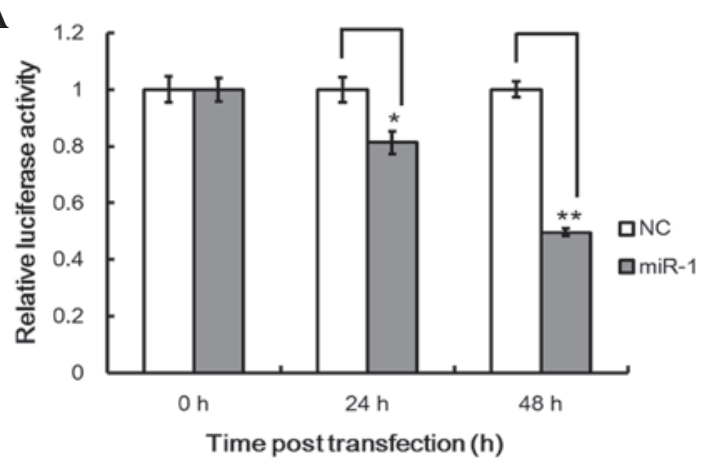

B

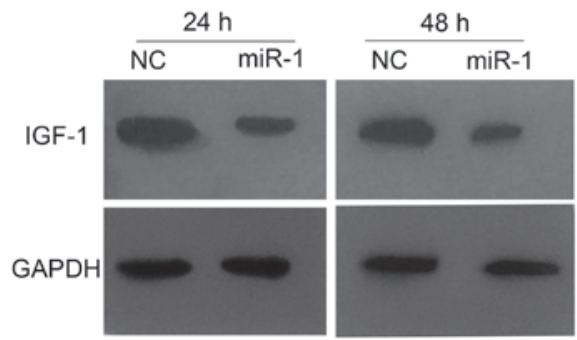

Figure 4. Insulin-like growth factor-1 (IGF-1) is a direct target of microRNA-1 (miR-1). (A) Relative luciferase activity analysis in cartilage cells. Sika deer cartilage cells were co-transfected with the recombination firefly luciferase reporter plasmid PmiR-RB-Report ${ }^{\mathrm{TM}}$-IGF-1-3'UTR and the negative scramble control (NC) or miR-1 mimic as indicated. Following 24 and $48 \mathrm{~h}$, the activity of Firefly luciferase was measured. Luciferase activity decreased compared with the control group following 24 and 48 h. (B) miR-1 inhibited the expression of the IGF-1 protein. The expression of IGF-1 protein and GAPDH in sika deer cartilage cells was detected using western blot analysis. Protein levels of IGF-1 and GAPDH decreased compared with the control group following 24 and 48 h, respectively. Data are presented as the mean \pm SD $(n=3)$. Similar results were found after three independent experiments. ${ }^{*} \mathrm{P}<0.05 ;{ }^{* *} \mathrm{P}<0.01 \mathrm{com}-$ pared with NC. into a luciferase reporter vector, and the effect of miR-1 on the luciferase activity of each construct in the cartilage cells was tested. As shown in Fig. 4A, miR-1 suppressed the luciferase activity of pmiR-IGF-1 by 50\% compared with the NCs $(\mathrm{P}<0.05)$. Western blot analysis demonstrated that the IGF-1 expression level decreased markedly compared with the NCs (Fig. 4B). Taken together, our results suggested that the 3'UTR of IGF-1 contains the miR-1 binding site and miR-1 is capable of inhibiting the expression of proteins with similar binding sites.

\section{Discussion}

miRNAs that are important in the regulation of gene expression have been discovered in various organisms. The main effect of miRNA is to silence the expression of target genes by complementary association with the 3'UTR on the mRNA of the target gene. A number of scholars hypothesize that there are thousands of miRNAs, and that these may control $\sim 30 \%$ of gene transcription in the human genome (20).

Numerous studies have confirmed that IGF-1, an important cell factor, rapidly improves the growth of deer antlers (3-5). Although the open reading frame (ORF) section of IGF-1 mRNA is widely conserved in all species, the 3'UTR section is not, such that the homology of Homo sapiens and the Chinese sika deer is $<80 \%$. Furthermore, the most effective part of the miRNA is known as the 'seed'. miRNA recognizes target mRNAs with seed-complementary sequences to direct post-transcriptional repression. Many downregulated targets against prediction algorithms have been developed based on this complementarity. This suggests that the miRNA seed sequence, which is the main regulating site, is necessary for 
regulating the expression of the target gene. The results also indicate that the 3'UTR of target gene, which is a complementary sequence of the miRNA seed region, may be evolutionarily well adapted various species.

However, there have been few reports with regard to Chinese sika deer miRNAs. In the present study, sequences for miR-1 of a known species were the same as those determined by TargetScan Human 5.1 Data Base analysis. Furthermore, the sequences of miR-1 from the total RNA of the Chinese sika deer corresponded with that of other species according to Affymetrix microRNA GeneChip analysis. This implies that miR-1 is widespread in different species and its sequence is conserved during evolution. We performed luciferase reporter screening in order to determine whether IGF-1 is targeted by miRNAs. We selected a pool of 25 miRNAs that had the potential to target IGF-1 according to the microRNA GeneChip, which is reported to have the best performance $(21,22)$. We found that miR-1 was capable of targeting the 3'UTR of IGF-1. Therefore, we proposed that the effect of miR-1 on IGF-1 expression results from their near-perfect complementarity, which leads to the degradation of IGF-1 mRNA. miR-1 expression profiles, available from the miRZ database (23), indicate that miR-1 is preferentially expressed in mesenchyme and cartilage cells (data not shown), suggesting that miR-1 may be involved in antler regeneration. This study provides initial evidence that miR-1 directly regulates the expression of IGF-1 by binding to the IGF-1 3'UTR, in order to promote antler growth and regeneration.

The results of this study showed that miR-1 affected the proliferation of antler cartilage cells. Transfection with an miR-1 mimic significantly inhibited the proliferation of cartilage cells compared with the NC group. These results suggested that a loss of miR-1 contributes to the promotion of cartilage cell proliferation. Furthermore, we found that the cell cycle distribution was affected by miR-1 expression in cartilage cells. The percentage of cells in the S-phase is an important indicator for determining the proliferation of cells. The smaller the percentage of cells in the S-phase, the lower the cell proliferation. FCM analysis indicated that the number of S-phase cells markedly decreased, and miR-1 transfection interacted with and disrupted the antler cells during mitosis. Therefore, this study provides evidence for the first time that miR-1 inhibits proliferation and cell cycle progression in the cartilage cells of deer antlers.

Furthermore, western blot analysis revealed that the expression levels of IGF-1 in cells transfected with the miR-1 mimic was lower than those in the NC group. This further illustrated the inhibitory effect of miR-1 on the expression of IGF-1. Sun et al (22) reported that miR-1 was able to regulate the expression of human IGF-1. In this study, miR-1 repressed the cellular mRNA and protein levels of IGF-1 by directly targeting the binding site within the 3'UTR, which subsequently led to a reduction in cell viability. These results suggested that miR-1 negatively regulates IGF-1 expression at the post-transcriptional level. The repression of the target by miRNA depends on the degree of complementarity between the miRNA and the target. In animals, mature miRNAs suppress gene expression by imperfect base pairing to the 3'UTR of the target mRNAs, leading to the repression of protein production and in certain cases, mRNA degradation.
Since the miRNA-mediated suppression of protein production occurs through a mechanism that operates following the initiation of protein synthesis, a reduction in protein production is not necessarily accompanied by a change in corresponding mRNA levels $(25,26)$.

The underlying mechanisms that mediate miR-1 deregulation in deer antler development remain elusive. This study suggests that miR-1 has a crucial role in antler regeneration. Since TargetScan predicted hundreds of potential targets of miR-1, and a single miRNA is capable of targeting multiple mRNAs in order to regulate gene expression (27), other miR-1 targets may also participate in antler development. Based on this theory, further studies are required to identify the additional roles of miR-1 in antler growth. In the future, we aim to determine whether the deregulation of miR-1 expression in antler growth is mediated by epigenetic alterations, including deregulated DNA methylation or histone modification.

\section{Acknowledgements}

This study was supported by a grant from the National Natural Science Foundation of China (no. 30972083) and was performed by the Jilin Provincial Science Technology Development Foundation of China (no. 20090574).

\section{References}

1. Miller SC, Bowman BM and Jee WS: Available animal models of osteopenia - small and large. Bone 17: S117-S123, 1995.

2. Kierdorf U, Li C and Price JS: Improbable appendages: deer antler renewal as a unique case of mammalian regeneration. Semin Cell Dev Biol 20: 535-542, 2009.

3. King GL, Kahn CR, Samuels B, et al: Synthesis and characterization of molecular hybrids of insulin and insulin-like growth factor I. The role of the A-chain extension peptide. J Biol Chem 257: 10869-10873, 1982.

4. Panagakos FS: Insulin-like growth factors-I and -II stimulate chemotaxis of osteoblasts isolated from fetal rat calvaria. Biochimie 75: 991-994, 1993.

5. Werther GA, Russo V, Baker N and Butler G: The role of the insulin-like growth factor system in the developing brain. Horn Res Paed 49: 37-40, 2004.

6. Zheng WH, Kar S, Dore S and Quirion R: Insulin-1ike growth factor-1 (IGF-1): a neuro protective trophic factor acting via the Akt kinase pathway. J Neural Transm Suppl 60: 261-272, 2000.

7. Rubin J, Ackert-Bicknell CL, Zhu L, et al: IGF-I regulates osteoprotegerin (OPG) and receptor activator of nuclear factor-kappaB ligand in vitro and OPG in vivo. J Clin Endocrinol Metab 87: 4273-4279, 2002.

8. Suttie JM, White RG, Breier BH and Gluckman PD: Photoperiod associated changes in insulin-like growth factor-I in reindeer. Endocrinology 129: 679-682, 1991.

9. Elliott JL, Oldham JM, Ambler GR, et al: Presence of insulin-like growth factor-I receptors and absence of growth hormone receptors in the antler tip. Endocrinology 130: 2513-2520, 1992.

10. Sadighi M, Haines SR, Skottner A, Harris AJ and Suttie JM: Effects of insulin-like growth factor-I (IGF-I) and IGF-II on the growth of antler cells in vitro. J Endocrinol 143: 461-469, 1994.

11. Li G, Gao X, Wang K, Gao Y and Zhao J: Research on the relationship of the serum concentrations of insulin growth factor 1 and the levels of nutrients in the sika deer and Chinese wapiti. Spec Wild Econ Anim Plant Res 26: 1-6, 2004.

12. Hu W, Meng X, Tian Y and Liu N: Cloning of a full-length cDNA encoding insulin-like growth factor I and its expression in antler tissue. J Northeast Forestry Univ 11: 71-75, 2011.

13. Shabalina SA and Spiridonov NA: The mammalian transcriptome and the function of non-coding DNA sequences. Genome Biol 5: $105,2004$.

14. Lee RC, Feinbaum RL and Ambros V: The C. elegans heterochronic gene lin-4 encodes small RNAs with antisense complementarity to lin-14. Cell 75: 843-854, 1993. 
15. Rajewsky N: MicroRNA target predictions in animals. Nat Genet 38: S8-S13, 2006.

16. Zeng Y: Principles of microRNA production and maturation. Oncogene 25: 6156-6162, 2006.

17. Tang F, Kaneda M, O' Carroll D, et al: Maternal microRNAs are essential for mouse zygotic development. Gen Dev 21: 644-648, 2007.

18. Stark A, Brennecke J, Bushati N, Russell RB and Cohen SM Animal microRNAs confer robusness to gene expression and have a significant on 3'UTR evolution. Cell 123: 1133-1146, 2005

19. Tay Y, Zhang J, Thoinson AM, Lim B and Rigoutsos I: MicroRNAs to Nanog, Oct4 and Sox 2 coding regions modulate embryonic stem cell differentiation. Nature 455: 1124-1128, 2008.

20. Tian S, Huang S, Wu S, Guo W, Li J and He X: MicroRNA-1285 inhibits the expression of p53 by directly targeting its 3 ' untranslated region. Biochem Biophys Res Commun 396 435-439, 2010.
21. Baek D, Villén J, Shin C, Camargo FD, Gygi SP and Bartel DP: The impact of microRNAs on protein output. Nature 455: 64-71, 2008.

22. Sun Y, Ge Y,Drnevich J,Zhao Y, Band M and Chen J: Mammalian target of rapamycin regulates miRNA-1 and follistatin in skeletal myogenesis. J Cell Biol 189: 1157-1169, 2010.

23. Hausser J, Berninger P, Rodak C, Jantscher Y, Wirth S and Zavolan M: MirZ: an integrated microRNA expression atlas and target prediction resource. Nucleic Acids Res 37: W266-W272, 2009.

24. Yang $M$ and Mattes J: Discovery, biology and therapeutic potential of RNA interference, microRNA and antagomirs. Pharmacol Ther 117: 94-104, 2008.

25. Nilsen TW: Mechanisms of microRNA-mediated gene regulation in animal cells. TRENDS Genet 23: 243-249, 2007.

26. Selbach M, Schwanhäusser B, Thierfelder N, Fang Z, Khanin R and Rajewsky N: Widespread changes in protein synthesis induced by microRNAs. Nature 455: 58-63, 2008. 